International Journal of Sustainable Agricultural Research

2020 Vol. 7, No. 4, pp. 255-266.

$\operatorname{ISSN}(e): 2312-6477$

$\operatorname{ISSN}(p):$ 2313-0393

DOI: $10.18488 /$ journal.70.2020.74.255.266

(C) 2020 Conscientia Beam. All Rights Reserved.

check for
updates

\title{
BREEDING FOR WEEVIL (Sitophilus Zeamais Motschulsky) RESISTANCE IN MAIZE (Zea mays $\mathrm{L}$ )
}

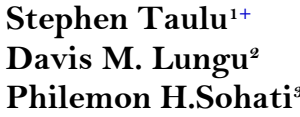

${ }^{\prime}$ David Livingstone College of Education, Livingstone, Zambia. Email:staulu@gmail.com Tel: +260977706485

${ }^{2, s}$ School of Agricultural Sciences, Department of Plant Science, University of Zambia.

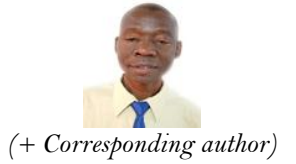

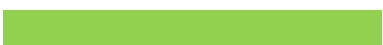

Article History

Received: 7 August 2020 Revised: 18 September 2020 Accepted: 30 September 2020 Published: 13 October 2020

\section{Keywords}

Resistance

Sitophilus zeamais

Zea mays

Susceptibility index
A study was conducted with an overall objective of establishing the determinants of weevil resistance in maize. Field experiments were done at GART using a North Carolina Design II with three replications and also at Nanga research in Mazabuka. Laboratory experiments were conducted at Zambia Agriculture Research Institute (ZARI) Entomology laboratory and University of Zambia, Food Science Department where insect bioassay and the biochemical tests were done respectively. In the insect bioassay grain hardness, grain weight loss, median development period, F1 progeny emergence and the Dobie index of susceptibility indices were measured. Protein and the Phenolic content were determined under the biochemical tests among genotypes. They were highly significant differences in all the twenty seven genotypes evaluated. Results showed that Parental survival accounted for $78.5 \%$ of the total variation, Phenolic content was strongly and positively correlated $\left(\mathrm{r}=0.423^{* * *}\right)$ with grain hardness providing a good measure of resistance accounting for the $10.9 \%$ of the total variation. The study showed the possibility of breeding maize genotypes with an increased resistance and also susceptible lines had a decreasing Phenolic content but increasing Phenolic content resulted in increased resistance. It was therefore concluded that Phenolic content and parental survival can be used as an indirect selection criteria for weevil resistance.

Contribution/Originality: This study is one of very few studies which have investigated the development of the Host-plant resistance as a pest control method is environmentally safe, economically cheaper method to farmers and most compatible with other components in the Integrated Pest Management initiatives.

\section{INTRODUCTION}

Maize, Zea mays L., is now one of the principal cereal food crops in the tropics and sub tropics (Makate, 2010). Maize is an essential component of the global food security and forms a major part of the diet of millions of people including Zambia (Kamanula et al., 2011). It grows under a wider range of ecological conditions depending on the variety (ASARECA-TUUSI, 2009). The crop is versatile in its use, environmental adaptation and it is also consumed all over the world by both humans and animals (Keba \& Waktole, 2013).Attempts have been made through breeding programmes for high yielding, as well field pests and disease resistant varieties which has resulted into many hybrids and composite varieties made available to farmers. Despite these high yielding varieties, farmers do not gain the maximum possible benefits from maize production if the quantity and the nutritional quality reduce (FAO, 1991). This is due to the various constraints including pest infestations at different levels of 
the production cycle particularly during storage phase. Storage losses due to pests threaten livelihoods of farmers across Africa (Kamanula et al., 2011). The maize weevil, $S$. zeamais is the most serious storage pest of maize in the tropics (Bosque-Perez \& Buddenhagen, 1992). The maize weevil affects the crop before harvest and multiplies further after storage (Caswell, 1962). Therefore development of the host-plant resistance as a pest control method is environmentally safe, economically cheaper to farmers and most compatible with other components in the integrated pest management (IPM) initiatives (Chapman, 2000). The overall objective of this study was to establish mechanisms of weevil resistance in maize, whose specific objectives were;

1. To characterize genotypes for traits related to weevil resistance in maize.

2. To estimate the genetic basis of the mechanisms of weevil resistance in maize.

\section{MATERIALS AND METHODS}

\subsection{Study Area}

A field experiment was set up at Golden Valley Agricultural Research Trust (GART) 80km north of Lusaka, Zambia during the 2011-2012 growing season in which hybridization was done. The field experiment was laid out using a North Carolina design II in which eight female lines from Zambia Agriculture Research Institute (ZARI) and nine male lines from CIMMYT were crossed. Plant emergence in some lines was poor due to poor synchronization and also due to the breakdown of the pump at Nanga during the critical periods of production; only twenty seven lines were successfully used for evaluation of weevil resistance.

\subsection{Laboratory Analysis}

Laboratory analysis was done in two ways: weevil bioassay and through biochemical bioassay. The laboratory analysis were done according to the procedures of Dobie (1977) and also (Makkar, 2003).

\subsection{Weevil Bioassay}

Laboratory analysis based on the weevil bioassay was done using the modified Dobie's method (Dobie, 1974; Serratos, Blanco-Labra, Arnason, \& Mihm, 1997). The freshly harvested seed of each variety were first sun dried to about $12-13 \%$ moisture content. Five cobs of each genotype were hand shelled and the grain was packed into $5 \mathrm{x} 8$ polythene bags and then they were closed by using the rubber bands and were then stored in a deep freezer at the temperature of $-16^{\circ} \mathrm{C}$ for one week to kill any previous infestation by the insects which included the adults, larva or eggs (Kossou, Mareck, \& Bosque-Perez, 1993).

Fifty (50) grams of the grains, from each plot was placed into new $350 \mathrm{ml}$ plastic jars, measuring $11.7 \mathrm{~cm}$ in height and about $5.2 \mathrm{~cm}$ in diameter at the mouth. The tops of the lids of these jars were cut out leaving only the screw top rings. The polythene screen lids allowing ventilation and preventing the escape of insects were placed on jars. Forty unsexed weevils of mixed age, in no choice experiments (Serratos et al., 1997) were initially counted into vials with the help of pairs of tweezers and a denominator Multiple - Tally counter were put into each jar.

Weevils were kept for ten (10) days for the $S$. zeamais to oviposit as described by Derera, Pixley, and Giga (2001). After 10 days sieving was done to remove adult weevils. Live and dead weevils were counted. Tweezers were used to probe immobile weevils to establish whether they were dead or alive. Progeny emergence counts were made every two days beginning 25 days after the removal of the parent insects and ending when all progeny $\left(\mathrm{F}_{1}\right)$ had emerged. Emerged progenies were removed from the jars at each count (Siwale, Mbata, McRobert, \& Lungu, 2007).

Seeds of each variety without the $S$. zeamais were kept under similar conditions and served as controls. The treatments were arranged in a Completely Randomized Design replicated three times. The jars were placed in the controlled temperature and the relative humidity room at $27-30^{\circ} \mathrm{C}$ temperature and 43 to $60 \%$ relative humidity. The relative humidity was provided by water placed in four troughs (Bekele \& Hassanali, 2001). The genotypes 
were kept undisturbed (except times of sieving the $F_{1}$ emergency) in the controlled temperature and relative humidity room for ninety days (90) days then assessment was done on physical parameters of seed as shown below.

\subsection{Grain Hardness}

Fifty grammes of each sample were weighed. Each sample was ground in a laboratory mill under the brand name of Retsh TM , Type ZM 1000 (GmbH \& Co. KG 5657 HAAN 1, Germany). The speed and the time setting were at 10000 revolutions per minute. The collected meal was put back in labelled plastic bags. The meal was then hand sifted. The collected flour and retained grit were emptied in separate labelled $5 \times 8 \mathrm{~cm}$ white plastics and these were subsequently weighed and data recorded.

The weight of the grit and flour were added together for each genotype to get the total weight, which was about the same weight as the original weight of grain from where flour and grit samples were derived. Grain hardness was expressed as percent grit of total weight of sample (grit plus flour after sieving a 50 g ground maize sample). Therefore grit percentage was used as a proxy for grain hardness.

\subsection{Seed Damage and Weight Loss}

Ninety (90) days after incubation, the glass jars were opened. The content in each jar was separated into grains, insects and dust using 4.7 and $1.0 \mathrm{~mm}$ sieves to assess each genotype's seed damage. The weight of dust produced was recorded. The damaged seeds (holed seeds) and the undamaged seed by the S.zeamais were counted. Seed damage was expressed as a proportion of the total number of seed sampled (Abebe, Tefera, Mugo, Beyene, \& Vidal, 2009). Seed weight loss was determined using the count and weight method (Gwinner, Harnisch, \& Muck, 1996).

Weight loss $(\%)=(\mathrm{Wu} \times \mathrm{Nd})-(\mathrm{Wd} \times \mathrm{Nu}) \times 100 / \mathrm{Wu} \times(\mathrm{Nd}+\mathrm{Nu})$;

Where $\mathrm{Wu}=$ Weight of undamaged seed.

$\mathrm{Nu}=$ Number of undamaged seed.

$\mathrm{Wd}=$ Weight of damaged seed.

$\mathrm{Nd}=$ Number of damaged seed.

\subsection{Dobie's Susceptibility Index}

The Dobie index was used as a criterion to separate varieties into different resistance groups. The Dobie Index of susceptibility was calculated using the formula

$\mathrm{I}=$ nlog (no of adult weevil progeny emerged)/MDP

Where I = Dobies's susceptibility index

MDP = median development period

The Dobies' index was used to separate maize genotypes into resistance or susceptibility groups following the scales used by CIMMYT (Pixley, 1997) which is as follows:

Dobie relative index of less than or equal to 4 was classified as resistant.

Dobie relative index of 4.1 to 6.0 classified as moderately resistant.

Dobie relative index of 6.1 to 8.0 classified as moderately susceptible.

Dobie relative index of 8.1 to 10 classified as susceptible.

Dobie relative index of more than 10 was classified as highly susceptible.

\subsection{Biochemical Bioassay}

\subsubsection{Protein Content}

The Kjeldahl procedure (Barbano, Lynch, \& Fleming, 1991) was used to determine the crude protein content. Twenty grams sample of the whole maize kernels were ground in a laboratory mill for each genotype. Three replications for each sample were used. 


\subsection{Phenolic Acid Content Determination}

This was determined by using the Folin - Ciocalteu method (Makkar, 2003) using the UV- Spectrophotometer. Tannic acid was used as a standard and this was done in three replications.

\subsection{Statistical Analysis}

The analysis of variance (ANOVA) for all the measured parameters was done using the GENSTAT Thirteen Edition and SPSS 16.0. Heritability was estimated using the North Carolina design II (Hallauer \& Miranda, 1988).

\section{RESULTS.}

\subsection{Protein Content}

Genotypes were significantly different $(\mathrm{p}<0.05)$ from each other for crude protein with an overall mean content of $8.8 \%$ as shown in Table 2. Among genotypes crude protein was in the range of $7.2 \%$ to $11.4 \%$. Genotypes $60 \mathrm{~N}$ and $24 \mathrm{~N}$ had high significant crude protein whose means were $11.4 \%$ and $10.6 \%$ respectively Table 2. Lower levels of crude protein were found in genotypes $95 \mathrm{U}(7.8 \%), 38 \mathrm{~N}(7.7 \%)$ and with the lowest in $78 \mathrm{~N}(7.4 \%)$ when compared to other genotypes Table 2 .

\subsection{Phenolic Content}

Genotypes were highly significantly different $(\mathrm{p}<0.05)$ for Phenolic content Table 1 . The Phenolic content among genotypes was found to be between 24 and $80.7 \mathrm{mg}$ per $100 \mathrm{~g}$ of maize grain. The mean Phenolic content among genotypes was $55.8 \mathrm{mg} / 100 \mathrm{~g}$ of maize grain. Genotype $60 \mathrm{~N}$ had the highest Phenolic content of $80.7 \mathrm{mg}$ per every $100 \mathrm{~g}$ of grain. The lowest level of phenolics was found in genotype $78 \mathrm{~N}$ (24mg per $100 \mathrm{~g}$ of grain).

\subsection{Parental Survival}

Parental survival of $S$. zeamais among the genotypes was significantly different $(\mathrm{p}<0.05)$. Table 2 presents the number of live adult weevils obtained after the ten days oviposition period. The overall mean survival number for parent weevils at the end of the oviposition period was 12.6. The number of parental survival among the entries was in the range of 3.0 to 32.7. The highest parental survival was recorded from genotype $78 \mathrm{~N}(32.7)$ which was not statistically different from $67 \mathrm{~N}(28.0)$. Least parental survival numbers were also found among the $31 \mathrm{~N}(3.0)$, $60 \mathrm{~N}$ (3.0) and $74 \mathrm{~N}(3.0)$ genotypes.

\subsection{Progeny Emergence}

The $\mathrm{F}_{1}$ progeny emergency among the genotypes was significantly different $(\mathrm{p}<0.05)$ among genotypes Table 1. The grand mean for progeny emergence was 15.5 and the range of emergence among entries was 2.3 to 98 weevils. Among all the maize genotypes evaluated, significantly higher numbers of $\mathrm{F}_{1}$ progenies (98) emerged from genotype $78 \mathrm{~N}$. Lowest numbers of $\mathrm{F}_{1}$ progenies emergence were found in genotypes $60 \mathrm{~N}(2.3), 74 \mathrm{~N}(3.0), 4 \mathrm{~N}(3.0)$ and $31 \mathrm{~N}(4.0)$.

\subsection{Median Development Period}

The median development period among the genotypes was significantly different among the genotypes $(p<$ 0.05). The median development period ranged from 26 to 79.8 days with the grand mean median development period of 50.3 days Table 2. Genotype $1 \mathrm{~N}$ had a statistically higher median development period of 79.8 days. Genotype $78 \mathrm{~N}$ had the least development period of 26.0 days. 
International Journal of Sustainable Agricultural Research, 2020, 7(4): 255-266

Table-1. Summary of the combined analysis of the analysis of variance of the measured traits in the maize genotypes.

\begin{tabular}{|c|c|c|c|c|c|c|c|c|c|c|c|}
\hline Source & $\overline{D F}$ & $\mathbf{S W}$ & GH & PS & $F_{1} E$ & MDP & MC & GWL & PR & $\mathbf{P H}$ & SI \\
\hline Entry & 26 & $0.006^{* * * *}$ & $21.82^{* * * *}$ & $21.2^{* * * *}$ & $1080.9^{* * * *}$ & $806.155^{* * * *}$ & $1.0635^{* *}$ & $25.43^{* * * *}$ & $6.323^{* * * *}$ & $6979.6^{* * * *}$ & $8.98^{* * * *}$ \\
\hline $\mathrm{CV}(\%)$ & & 2.4 & 6.3 & 7.4 & 5.5 & 1.6 & 4.7 & 3.5 & 3.1 & 4.2 & 8.4 \\
\hline S.E.D & & 0.0073 & 2.171 & 0.748 & 0.6789 & 0.754 & 0.6719 & 0.1592 & 0.2167 & 2.187 & 0.1414 \\
\hline
\end{tabular}

Note: **** = significant at $5 \%$ level

Key: $\mathrm{SW}=1$ 10oseed weight, $\mathrm{GH}=$ grain hardness, $\mathrm{PS}=$ Parental survival, $\mathrm{F}_{1} \mathrm{E}=$ progeny emergency, $\mathrm{MDP}=$ median development period, $\mathrm{MC}=$ moisture content, $\mathrm{GWL}=$ grain weight loss, $\mathrm{PR}=$ protein, $\mathrm{PH}=$ Phenolic, SI = susceptibility index 
Table-2. Summary of means for the genotypes.

\begin{tabular}{|c|c|c|c|c|c|c|c|c|c|c|}
\hline Entry & MDP & PS & $\mathbf{F}_{1}$ & PH & GWL & $\mathrm{MC}$ & PR & SI & GH & $100 \mathrm{SW}$ \\
\hline $1 \mathrm{~N}$ & 79.84 & 4.00 & 4.00 & 80.00 & 4.53 & 11.60 & 9.23 & 0.75 & 47.83 & 35.24 \\
\hline $4 \mathrm{~N}$ & 78.10 & 8.67 & 3.00 & 78.67 & 4.60 & 12.57 & 9.57 & 0.61 & 45.84 & 35.44 \\
\hline $77 \mathrm{~N}$ & 63.10 & 10.00 & 9.00 & 58.00 & 4.67 & 13.23 & 9.30 & 1.65 & 39.71 & 30.25 \\
\hline $60 \mathrm{~N}$ & 59.45 & 3.00 & 2.33 & 80.67 & 4.27 & 12.40 & 11.37 & 0.37 & 51.67 & 35.44 \\
\hline $8 \mathrm{~N}$ & 52.30 & 18.33 & 29.00 & 68.33 & 6.93 & 11.77 & 9.33 & 2.14 & 39.24 & 36.02 \\
\hline $6 \mathrm{~N}$ & 56.41 & 11.00 & 15.00 & 73.00 & 4.80 & 12.60 & 10.23 & 1.61 & 43.29 & 36.27 \\
\hline $74 \mathrm{~N}$ & 78.14 & 3.00 & 3.00 & 58.00 & 2.47 & 13.43 & 9.20 & 0.82 & 43.07 & 36.66 \\
\hline $24 \mathrm{~N}$ & 62.72 & 4.33 & 4.00 & 75.33 & 4.93 & 12.83 & 10.60 & 0.80 & 47.49 & 33.83 \\
\hline $26 \mathrm{~N}$ & 31.00 & 29.00 & 27.00 & 31.00 & 6.73 & 12.20 & 7.57 & 4.62 & 34.50 & 30.87 \\
\hline $73 \mathrm{U}$ & 52.45 & 21.00 & 10.33 & 66.33 & 4.67 & 12.70 & 9.60 & 1.53 & 38.04 & 38.30 \\
\hline $31 \mathrm{~N}$ & 69.72 & 3.00 & 4.00 & 70.67 & 4.13 & 12.60 & 7.87 & 0.85 & 45.28 & 38.47 \\
\hline $67 \mathrm{~N}$ & 29.04 & 28.00 & 31.00 & 29.00 & 7.13 & 12.93 & 8.70 & 5.14 & 38.17 & 42.87 \\
\hline $19 \mathrm{~N}$ & 71.43 & 4.00 & 5.00 & 51.00 & 5.13 & 11.77 & 9.00 & 1.37 & 39.13 & 36.66 \\
\hline $56 \mathrm{~N}$ & 53.65 & 4.33 & 4.67 & 58.00 & 4.13 & 11.80 & 9.23 & 1.15 & 37.67 & 45.54 \\
\hline $13 \mathrm{~N}$ & 29.00 & 12.33 & 21.33 & 62.00 & 4.67 & 11.60 & 7.17 & 2.14 & 40.82 & 35.40 \\
\hline $45 \mathrm{U}$ & 41.35 & 9.67 & 8.00 & 73.67 & 4.33 & 13.47 & 8.47 & 1.23 & 37.80 & 43.10 \\
\hline $80 U$ & 46.35 & 18.33 & 30.00 & 47.00 & 7.00 & 12.67 & 8.20 & 3.14 & 43.22 & 38.50 \\
\hline $66 \mathrm{U}$ & 45.18 & 13.33 & 10.67 & 66.33 & 6.47 & 13.30 & 9.00 & 1.55 & 43.07 & 39.65 \\
\hline $46 \mathrm{U}$ & 45.90 & 8.67 & 6.00 & 67.33 & 4.87 & 12.47 & 8.80 & 1.16 & 41.67 & 43.60 \\
\hline $63 \mathrm{U}$ & 57.14 & 8.00 & 14.67 & 46.00 & 4.73 & 12.77 & 9.23 & 2.54 & 43.17 & 38.67 \\
\hline $91 \mathrm{U}$ & 48.55 & 7.00 & 7.00 & 45.33 & 4.93 & 13.87 & 8.37 & 1.86 & 38.50 & 38.47 \\
\hline $12 \mathrm{~N}$ & 26.89 & 22.00 & 20.00 & 32.00 & 7.40 & 12.77 & 7.83 & 4.07 & 38.60 & 34.87 \\
\hline $38 \mathrm{~N}$ & 37.34 & 20.67 & 5.33 & 28.00 & 5.60 & 12.87 & 7.73 & 4.20 & 39.13 & 35.25 \\
\hline $78 \mathrm{~N}$ & 26.00 & 32.67 & 98.00 & 24.00 & 19.07 & 12.10 & 7.40 & 8.30 & 33.78 & 41.10 \\
\hline $95 \mathrm{U}$ & 33.28 & 19.67 & 23.67 & 33.00 & 4.60 & 13.60 & 7.80 & 4.16 & 40.37 & 40.87 \\
\hline $80 \mathrm{~N}$ & 40.11 & 10.33 & 9.00 & 53.00 & 4.47 & 12.50 & 8.40 & 1.80 & 38.50 & 32.00 \\
\hline $74 \mathrm{U}$ & 56.03 & 5.33 & 5.00 & 50.00 & 5.20 & 12.00 & 9.10 & 1.40 & 41.13 & 38.63 \\
\hline Mean & 50.26 & 12.58 & 15.18 & 55.76 & 5.65 & 12.61 & 8.82 & 2.26 & 41.14 & 37.4 \\
\hline LSD & 1.482 & 1.513 & 1.848 & 7.85 & 0.316 & 0.133 & 0.433 & 0.276 & 1.748 & 0.014 \\
\hline
\end{tabular}

\subsection{Grain Hardness}

Grain hardness showed discrimination among genotypes. They were significant differences among the genotypes $(\mathrm{p}<0.05)$. Grand mean hardness value of $41.1 \%$ was observed among genotypes. Genotype $60 \mathrm{~N}$ was statistically higher than the other genotypes with $51.7 \%$ and genotype $1 \mathrm{~N}$ had similarly a higher grain hardness value of $47.8 \%$. Genotypes $26 \mathrm{~N}(34.5 \%)$ and $78 \mathrm{~N}(33.8 \%)$ had statistically lower grain hardness values.

Table-3. Correlation coefficients of $S$. zeamais infestation of the maize genotypes.

\begin{tabular}{|c|c|c|c|c|c|c|c|c|c|}
\hline PAR & SI & $\mathbf{F}_{1} \mathbf{E}$ & MDP & GWL & GH & $100 \mathrm{SW}$ & PS & PH & PR \\
\hline SI & 1 & & & & & & & & \\
\hline $\mathrm{F}_{1} \mathrm{E}$ & 0.197 & 1 & & & & & & & \\
\hline MDP & -0.312 & -0.082 & 1 & & & & & & \\
\hline GWL & -0.308 & 0.083 & 0.189 & 1 & & & & & \\
\hline $\mathrm{GH}$ & $-0.361^{* * * *}$ & 0.085 & $-0.355^{* * * *}$ & -0.131 & 1 & & & & \\
\hline $100 \mathrm{SW}$ & $-0.473^{* * * *}$ & 0.093 & 0.171 & 0.167 & -0.138 & 1 & & & \\
\hline PS & $0.612^{* * *}$ & -0.316 & $-0.504^{* * * *}$ & 0.248 & -0.122 & 0.052 & 1 & & \\
\hline $\mathrm{PH}$ & -0.213 & -0.048 & -0.27 & 0.09 & $0.423^{* * *}$ & 0.14 & -0.225 & 1 & \\
\hline PR & -0.155 & 0.172 & -0.289 & -0.151 & -0.042 & 0.06 & -0.095 & -0.144 & 1 \\
\hline
\end{tabular}

Key: SI =susceptibility index, F1 E = F1 emergency, MDP = median development period, GWL = grain weight loss, $100 \mathrm{SW}=100$ seed weight, PS = parental survival, $\mathrm{PH}=$ Phenolic, $\mathrm{PR}=$ proteins, $\mathrm{PAR}=$ parameter.

\subsection{Dobie Index of Susceptibility (SI)}

Significant differences $(\mathrm{p}<0.05)$ were observed on index of susceptibility among the genotypes evaluated

Table 1. The SI in this study ranged from 0.4 to 8.3 for genotypes $60 \mathrm{~N}$ and $78 \mathrm{~N}$ respectively. According to the 
CIMMYT classification, out of the twenty seven maize genotypes evaluated against $S$. zeamais for resistance, twenty three genotypes were found to be relatively resistant; three genotypes $26 \mathrm{~N}, 12 \mathrm{~N}$ and $67 \mathrm{~N}$ were moderately resistant. Only one genotype $(78 \mathrm{~N})$ was moderately susceptible.

The results Table 3 shows an inverse relationship between the susceptibility index (SI) and median development period, grain weight loss (\%), grain hardness, 100 seed weight, Phenolic and protein. The parental survival $\left(\mathrm{r}=0.612^{* * *}\right)$ and $\mathrm{F}_{1}$ progeny emergencies $(\mathrm{r}=0.197)$ were positively correlated to susceptibility index. The inter component correlations among traits showed that median development period was positively correlated with grain weight $\operatorname{loss}(\mathrm{r}=0.189), 100$ seed weight $(\mathrm{r}=0.171)$ though they were not significant $(\mathrm{p}>0.05)$. Seed weight was also positively correlated to grain weight loss $(r=0.167)$ but it was not $\operatorname{significant}(p>0.0$.

\subsection{Stepwise Multiple Regression}

Significant contributions to the total variation were observed from the four traits namely parental survival of adult weevils, Phenolic content, $F_{1}$ progeny emergency and grain hardness. Parental survival had a most significant influence on susceptibility index of $78.5 \%$ of the total variation Table 4 . Additional of other variables such as Phenolic, $\mathrm{F}_{1}$ progeny emergency, and grain hardness also showed significant influence of $10.9 \%, 8 \%$ and $0.5 \%$ respectively.

Table-4. Stepwise correlation of susceptibility index and other traits.

\begin{tabular}{|c|c|c|c|c|}
\hline Variable & Partial square & R-model square & R-F value & $\operatorname{Pr}>\mathbf{F}$ \\
\hline Parental survival & 0.785 & 0.785 & 91.452 & 0.000 \\
\hline Phenolics & 0.109 & 0.894 & 24.589 & 0.000 \\
\hline $\mathrm{F}_{1}$ emergency & 0.08 & 0.974 & 71.874 & 0.000 \\
\hline Grain hardness & 0.005 & 0.979 & 5.584 & 0.000 \\
\hline
\end{tabular}

Table-5. Classification of Maize weevil resistance among genotypes using Dobie Index.

\begin{tabular}{|c|c|c|}
\hline Entry & Dobie Index & Classification \\
\hline $1 \mathrm{~N}$ & 0.75 & Resistant \\
\hline $4 \mathrm{~N}$ & 0.59 & Resistant \\
\hline $77 \mathrm{~N}$ & 1.28 & Resistant \\
\hline $60 \mathrm{~N}$ & 0.48 & Resistant \\
\hline $8 \mathrm{~N}$ & 2.14 & Resistant \\
\hline $6 \mathrm{~N}$ & 1.61 & Resistant \\
\hline $74 \mathrm{~N}$ & 0.8 & Resistant \\
\hline $24 \mathrm{~N}$ & 0.8 & Resistant \\
\hline $73 \mathrm{U}$ & 1.53 & Resistant \\
\hline $31 \mathrm{~N}$ & 0.84 & Resistant \\
\hline $67 \mathrm{~N}$ & 5.14 & Moderately resistant \\
\hline $19 \mathrm{~N}$ & 1.37 & Resistant \\
\hline $56 \mathrm{~N}$ & 1.14 & Resistant \\
\hline $13 \mathrm{~N}$ & 2.14 & Resistant \\
\hline $45 \mathrm{U}$ & 1.23 & Resistant \\
\hline $80 \mathrm{U}$ & 3.14 & Resistant \\
\hline $66 \mathrm{U}$ & 1.55 & Resistant \\
\hline $46 \mathrm{U}$ & 1.16 & Resistant \\
\hline $63 \mathrm{U}$ & 1.71 & Resistant \\
\hline $91 \mathrm{U}$ & 1.86 & Resistant \\
\hline $12 \mathrm{~N}$ & 4.07 & Moderately resistant \\
\hline $38 \mathrm{~N}$ & 1.4 & Resistant \\
\hline $78 \mathrm{~N}$ & 8.34 & Moderately susceptible \\
\hline $95 \mathrm{U}$ & 2.88 & Resistant \\
\hline $80 \mathrm{~N}$ & 1.8 & Resistant \\
\hline $74 \mathrm{U}$ & 1.29 & Resistant \\
\hline $26 \mathrm{~N}$ & 4.62 & Moderately resistant \\
\hline
\end{tabular}




\subsection{Genetic Parameters}

It was observed that the non additive variation controls all the traits that were measured in this study Table 7. The calculated narrow sense heritability was low in most traits ranging from 5.9 to 22.2\% Table 6. Heritability analysis according to Derera et al. (2001) on genetic analyses can be performed only for the index of susceptibility because it incorporates all resistance parameters. Therefore in this study it was estimated that heritability of the traits was $20.9 \%$ since this was the heritability based on susceptibility index in this study Table 6 . The heritability estimate was considered low since it was below $30 \%$.

Table-6. Estimated genetic parameters for some traits in the maize genotypes.

\begin{tabular}{c|c|c|c|c|c|c|c|c}
\hline \multicolumn{1}{c|}{ Table-6. Estimated genetic parameters for some traits in the maize genotypes. } \\
$\begin{array}{c}\text { Variance } \\
\text { Component }\end{array}$ & $\mathbf{F}_{1} \mathbf{E}$ & MDP & $\begin{array}{c}\text { Grain } \\
\text { weight loss }\end{array}$ & Hardness & $\begin{array}{c}\text { Kernel } \\
\text { weight }\end{array}$ & Phenols & Proteins & $\begin{array}{c}\text { Susceptibility } \\
\text { Index }\end{array}$ \\
\hline$\sigma_{\mathrm{Am}}{ }^{2}$ & 157.36 & 542.2 & 2.69 & 38.76 & 0.000025 & 107.2 & 0.156 & 3.756 \\
\hline$\sigma_{\mathrm{Af}}{ }^{2}$ & -23.52 & -174.8 & 0.296 & 13.72 & 0.00114 & -40 & -0.4753 & -0.648 \\
\hline$\sigma_{\mathrm{T}}{ }^{2}$ & 66.9 & 183.68 & 1.496 & 26.24 & 0.000582 & 33.6 & 0.3318 & 1.554 \\
\hline$\sigma_{\mathrm{D}}{ }^{2}$ & 239 & 812.2 & 3.752 & 68.68 & 0.006 & 39.76 & 5.53 & 4.3 \\
\hline $\mathrm{h}^{2}(\%)$ & 17.9 & 15.6 & 22.2 & 21.65 & 8.12 & 8.3 & 5.9 & 20.9 \\
\hline
\end{tabular}

Table-7. Summary for the average degree of dominance for the traits.

\begin{tabular}{c|c}
\hline Trait & Degree of dominance \\
\hline $\mathrm{F}_{1}$ emergence & 1.74 \\
\hline Median development period & 1.73 \\
\hline Grain weight loss & 1.67 \\
\hline Grain hardness & 1.88 \\
\hline Mortality $(\%)$ & 1.69 \\
\hline Parental survival & 2.80 \\
\hline Phenolic content & 6.33 \\
\hline Protein content & 8.67
\end{tabular}

Table-8. Mean squares for susceptibility index and the other agronomic traits among the parental lines.

\begin{tabular}{|c|c|c|c|c|c|c|c|c|c|}
\hline Source of Variation & df & MDP & PS & F1 & $\mathbf{P H}$ & GWL & PR & $\mathbf{G H}$ & SI \\
\hline Rep & 2 & 0.79 & 0.3 & 0.28 & 4.12 & 10.76 & 9.16 & 3.26 & 0.16 \\
\hline Crosses & 15 & $0.21^{*}$ & $0.28^{*}$ & 1.87 & $22.65^{* *}$ & $8.7^{*}$ & 4.61 & $2.87^{* *}$ & $0.41^{* * *}$ \\
\hline GCA males & 3 & 0.68 & 0.23 & 0.89 & $25.46^{* *}$ & $6.8^{*}$ & 3.55 & $1.51^{*}$ & $0.58 *$ \\
\hline GCA females & 3 & 0.26 & 0.17 & 0.55 & $11.71^{* *}$ & $3.9^{* *}$ & 3.33 & $2.86^{* *}$ & $0.56^{*}$ \\
\hline SCA & 9 & 0.09 & 3.55 & 0.08 & $12.41 *$ & $4.1 * *$ & 1.81 & 2.14 & 0.35 \\
\hline Error & 30 & 0.55 & 0.99 & 3.61 & 3.29 & 0.34 & 4.61 & 4.12 & 1.4 \\
\hline $\mathrm{CV} \%$ & & 1.5 & 2.2 & 3.4 & 4.6 & 3.2 & 2.6 & 3.8 & 3.6 \\
\hline
\end{tabular}

Note: *, ** significant at $5 \%$ and $1 \%$ probability level respectively.

Table-9. SCA effects.

\begin{tabular}{|c|c|c|c|c|c|c|c|c|c|c|}
\hline Entry & CROSS & MDP & PS & F1 & PH & GWL & PR & SI & GH & $100 \mathrm{SW}$ \\
\hline $1 \mathrm{~N}$ & $151 \times 10075$ & $29.58^{* *}$ & -8.58 & $-11.18^{* * *}$ & $24.24^{* * *}$ & -1.12 & 0.7 & -1.51 & $6.69^{* *}$ & $-2.16^{*}$ \\
\hline $67 \mathrm{~N}$ & $151 \times 10096$ & -34.45 & $15.42^{* * *}$ & $15.82^{* * *}$ & $-26.76^{* *}$ & $1.48^{*}$ & 0.17 & $2.88^{*} *$ & -2.97 *** & $5.47 * *$ \\
\hline $74 \mathrm{U}$ & $151 \times 10111$ & $5.77 *$ & -7.25 & $-10.18 * *$ & -5.76 & -0.45 & 0.57 & -0.86 & -0.01 & 1.23 \\
\hline $60 \mathrm{~N}$ & $151 \times 10112$ & $9.19^{* *}$ & -9.58 & $-12.85^{*} *$ & $24.91^{*} * *$ & -1.38 & $2.84^{*} * *$ & $-1.89^{*} *$ & $10.53^{*} *$ & -1.96 \\
\hline $74 \mathrm{~N}$ & $152 \times 10075$ & $27.88^{*} *$ & -9.58 & $-12.18 * *$ & 2.24 & -3.18 & 0.67 & -1.44 & 1.93 & -0.74 \\
\hline $56 \mathrm{~N}$ & $152 \times 10096$ & 3.39 & -8.25 & $-10.51 * *$ & 2.24 & -1.52 & 0.7 & -1.11 & -3.47 & $8.14^{*}$ \\
\hline $63 \mathrm{U}$ & $152 \times 10111$ & $6.88 *$ & -4.58 & -0.51 & -9.76 & -0.92 & 0.7 & 0.28 & 2.03 & 1.27 \\
\hline $38 \mathrm{~N}$ & $152 \times 10112$ & -12.92 & 8.09 *** & $-9.85^{* * *}$ & $-27.76^{* *}$ & -0.05 & $-2.8 * *$ & 1.94 & -2.01 & -2.15 \\
\hline $24 \mathrm{~N}$ & $1212 \times 10075$ & $12.46^{* *}$ & $-8.25^{* *}$ & $-11.18^{* *}$ & 19.57 *** & -0.72 & 2.07 *** & -1.46 & 6.35 & -3.57 ** \\
\hline $12 \mathrm{~N}$ & $1212 \times 10096$ & -23.37 *** & $9.42^{*} *$ & 4.82 & $-23.76^{* * *}$ & 1.75 & -0.7 & 1.81 & -2.54 & $-2.53^{*}$ \\
\hline $77 \mathrm{U}$ & $1212 \times 10111$ & $12.84^{* * *}$ & -2.58 & $-6.18^{* * *}$ & 2.24 & -0.98 & 0.77 & -0.61 & -1.43 & $-7.15^{*}$ \\
\hline $91 \mathrm{U}$ & $1212 \times 10112$ & -1.71 & $-5.58 *$ & $-8.18^{* * *}$ & -10.43 & -0.72 & -0.16 & -0.4 & -2.64 & 1.07 \\
\hline $6 \mathrm{~N}$ & $917 \times 10075$ & $6.15^{* *}$ & -1.58 & -0.18 & 17.24 & $-0.85^{*}$ & 1.7 & -0.65 & 2.15 & -1.13 \\
\hline $80 \mathrm{U}$ & $917 \times 10096$ & -3.91 & $5.75^{* * *}$ & $14.82^{* * *}$ & -8.76 & 1.35 & -0.33 & 0.88 & 2.08 & 1.1 \\
\hline $4 \mathrm{~N}$ & $917 \times 10111$ & $27.84^{* *}$ & -3.91 & $-12.18^{* *}$ & $22.91^{*} *$ & -1.05 & 1.04 & -1.65 & 4.7 & $-1.96^{*}$ \\
\hline $80 \mathrm{~N}$ & $917 \times 10112$ & $-10.15^{* * *}$ & -2.25 & $-6.18^{* * *}$ & $-2.76^{*}$ & -1.18 & -0.13 & -0.46 & -2.64 & $-5.4^{* *}$ \\
\hline s.e.d & & 0.56 & 0.399 & 0.861 & 0.319 & 0.2232 & 0.1361 & 0.1211 & 0.67 & $\mathrm{O}$ \\
\hline
\end{tabular}


Table-10. GCA effects for the parental lines.

\begin{tabular}{|c|c|c|c|c|c|c|c|}
\hline Males & F1 & GWL & SI & MDP & $\mathbf{P H}$ & PR & PS \\
\hline 10096 & $-7.75 * *$ & $-6.88 * *$ & $-2.63 * *$ & $24.54 * *$ & $29.69 * *$ & $1.06 * *$ & $-6.50 * *$ \\
\hline 10112 & $3.91^{* * *}$ & $-3.95^{* *}$ & $-1.62 * *$ & $9.54^{* *}$ & $40.79^{* *}$ & $-0.30^{*}$ & $-14.16^{* * *}$ \\
\hline s.e.d & 0.84 & 0.21 & 0.08 & 0.5 & 0.28 & 0.126 & 0.38 \\
\hline 151 & $2.25^{* *}$ & $4.62 * *$ & $2.17 * *$ & $-28.79 * *$ & $-29.74 * *$ & $-0.47 *$ & $12.90^{* * *}$ \\
\hline 152 & $3.91^{* * *}$ & $6.12^{* *}$ & $2.51 * *$ & $-30.46^{* *}$ & $-27.51 * *$ & $-0.87 * *$ & 7.37 ** \\
\hline 917 & $5.91 * *$ & $5.75^{* *}$ & $2.20 * *$ & $-27.46^{* *}$ & $-28.11^{*} *$ & $-0.77 *$ & $18.44 * *$ \\
\hline 1212 & $3.58^{* *}$ & $6.75^{* *}$ & $2.89^{* *}$ & $-29.46^{* * *}$ & $-30.84^{* * *}$ & $-0.87^{*}$ & $7.37^{* *}$ \\
\hline s.e.d & 0.861 & 0.2232 & 0.1211 & 0.56 & 0.319 & 0.1361 & 0.3991 \\
\hline
\end{tabular}

\subsection{Combining Ability}

The mean squares due to general combining ability were significant for all variables measured. However, the mean squares due to the specific combining ability were not significant for the same traits Table 8 .

Estimates of the Specific Combining Ability (SCA) and the General Combining Ability (GCA) effects for the various traits are presented in Table 9 and 10 respectively.

\section{DISCUSSION}

The results were discussed in two parts.

\subsection{Factors Related to Weevil Resistance in Maize}

Protein content was negatively correlated with the susceptibility index of maize genotypes. This was consistent with findings reported by Dobie (1977); Keba and Waktole (2013). Furthermore, genotypes in this study with higher protein content were classified to be resistant based on CIMMYT (2001) classification. This was evident in genotype $60 \mathrm{~N}$ which had the highest protein content $(11.3 \%)$ and the number of adult weevils surviving at the end of the experiment on this genotype was only 3.0. The lowest genotypes in terms of protein content were genotypes $95 \mathrm{U}(7.8 \%)$ and $78 \mathrm{~N}(7.4 \%)$ which had parental survival numbers of 19.7 and 32.7 respectively. This was also consistent with what other researchers found out (Derera et al., 2001; García-Lara et al., 2004).

Further analysis with the stepwise regression analysis which is a stronger tool than correlation for use in indirect selection showed that protein content was not significant in the observed susceptibility index. This suggests that none of the maize varieties tested was completely resistant for proteins. These findings were consistent with Siwale et al. (2007); Tongjura, Amuga, and Mafuyai (2010). Although protein may seem to have some antibiosis effect, lack of a definite relationship with physical resistance parameters in this study may indicate other resistance factors in maize studied.

Arnason et al. (1997) also reported on the presence of biochemical compounds, Phenolics especially the ferulic acid in the maize grain in conferring resistance. The level of the Phenolic compounds was negatively correlated with susceptibility index. This was in agreement with the findings reported by Dobie (1977). It was also noticed that genotypes with the highest amount of the phenols like genotype $60 \mathrm{~N}(80.676 \mathrm{mg} / 100 \mathrm{~g})$ had less grain weight loss (4.3\%) since weevil attack may have been prevented by the amounts of Phenolic compounds particularly ferulic acid component. Genotypes with lower amounts of Phenolic, $78 \mathrm{~N}$, had $24 \mathrm{mg} / 100 \mathrm{~g}$ of grain had a higher grain weight loss of $19 \%$. This was also consistent with other authors Derera et al. (2001); Classen et al. (1990); Sen, Mukhopadhyay, Wetzel, and Biswas (1994); Arnason et al. (1997). These authors reported that phenolic compounds particularly ferulic acid had an influence on the hardness of the grain such that it was able to make the cell walls hard and limit the biodegradability of the cell wall polysaccharides by insects. The Phenolic acids were able to cause 
adverse effects to weevil feeding behavior and survival. Therefore, biochemical screening of the maize grain may be used as a first step towards selection of genotypes for resistance.

In terms of grain weight loss, resistant maize varieties had a minimum grain damage and small quantity of powder formed. Grain weight loss was highest in genotype $78 \mathrm{~N}$ in which there was a $19 \%$ grain weight loss. The median development period among the genotypes had an average of 50.3 days. The range of the median development period was wider (26 to 79.8 days). The period was longer in the resistant genotype $(60 \mathrm{~N})$ in which the median development period was 79.8 days but median development period was shorter in the susceptible genotype $78 \mathrm{~N}$ with median development period of 26 days. For susceptible genotypes the development period of weevils was shorter and vice versa.

Higher grain weight loss values may have been expected in this study if the young weevils of same age particularly o to 3 weeks old were used. This was demonstrated by Dobie (1977) in which fecundity and the feeding of maize was highest when weevils were in the range of 0 to 3 weeks old after which there was a steady decline. In this study, weevils which were used were of unknown age such that it was possible that some of the weevils used may have been past the 0 to 3 weeks old. Parental survival was negatively and significantly $(p<0.05)$ correlated to the median developmental period $(\mathrm{r}=-0.504 * * *)$. Through stepwise multiple regression analysis, it was observed that parental survival in terms of explaining total variation had a highest contribution of $78.5 \%$. This means that the number of parent weevils that were alive or dead in given genotypes gave an indication of susceptibility or resistance. There was a negative correlation $(r=-0.355)$ between grain hardness and the median development period of the weevils which was significant $(\mathrm{p}<0.05)$. This means that genotypes with a harder testa took more time for the weevils to develop on the grain as was evident in the low susceptibility index value indicating resistant genotypes Table 5. Susceptible genotype like genotype $78 \mathrm{~N}$ with low grain hardness $(33.8 \%)$ had 98 progenies Table 2 emerging indicating a high possibility of higher damage by weevils.

Grain hardness was further found to be significantly $(\mathrm{p}<0.05)$ and positively associated with Phenolic compounds $\left(\mathrm{r}=0.423^{* * *}\right)$. Grain hardness contributed $0.5 \%$ to the total variation of susceptibility index. Increased Phenolic compounds increased hardness as well which may have contributed to the resistance of genotypes. This was consistent with the study reported by Arnason et al. (1997) in which increased Phenolic content was observed to be concentrated on the cell walls of the grain and then makes the grain harder depending on the concentration of Phenolic content.

The range of susceptibility index values obtained in this study ranged from 0.4 for genotype $60 \mathrm{~N}$ to 8.4 for genotype $78 \mathrm{~N}$. Most genotypes in this study were resistant.

\subsection{Inheritance of Weevil Resistance in Maize}

The study showed that narrow sense heritability calculated was low $(20.9 \%)$, indicating a very low gain to selection Table 6. To breed for higher Phenolic content among genotypes in a breeding programme it would be necessary so that to do the population improvement through recurrent selection since the trait has the heterotic response. This means that inbred lines will have to be developed in order to come up with hybrids. These hybrids will express heterosis in terms of high weevil resistance. The negative GCA effects of the female inbred lines indicate reduced Phenolic content in the maize results into the increased positive significant susceptibility values. This indicates that female lines are likely to contribute an increased weevil attack in their crosses. Through indirect selection of some of these traits like Phenolic, it is possible to improve maize varieties to weevil attack. It is possible that the contribution of the Phenolic compounds could have been more than $10.9 \%$ Table 4 if parental lines used would have had a higher Phenolic content than the mean Phenolic content of $36.8 \mathrm{mg} / 100 \mathrm{~g}$ of grain Table 2 . Female line 151 and male line 10112 could be used as parents in making synthetic populations for recurrent selection. While doing the SCA effects among genotypes for yield, testing for weevil resistance among hybrids can 
be done because this trait is showing some heterotic response in this study. This is in agreement with the study that was conducted by Serratos et al. (1993).

Funding: This study received no specific financial support.

Competing Interests: The authors declare that they have no competing interests.

Acknowledgement: All authors contributed equally to the conception and design of the study.

\section{REFERENCES}

Abebe, F., Tefera, T., Mugo, S., Beyene, Y., \& Vidal, S. (2009). Resistance of maize varieties to the maize weevil Sitophilus zeamais (Motsch.)(Coleoptera: Curculionidae). African Journal of Biotechnology, 8(21), 5937-5943.Available at: https://doi.org/10.5897/ajbo9.821.

Arnason, T., Conilth de Beyssac, B., Philogene, B. J. R., Bervinson, D., Serratos, J. A., \& Mihm, J. A. (1997). Mechanisms of resistance in maize grain to the maize weevil and the larger grain borer. In Mihm, J.A (Ed.), Insect Resistant maize - recent advances and utilization. Paper presented at the International Symposium., Mexico City. 27 Dec -3 Dec 1994. CIMMYT, Mexico City.

ASARECA-TUUSI. (2009). Drought and low-N torelant maize germplasm and varieties- inbred lines, populations, OPVs, Hybrids.

Barbano, D. M., Lynch, J. M., \& Fleming, J. R. (1991). Direct and indirect determination of true protein content of milk by Kjeldahl analysis: Collaborative study. Journal of the Association of Official Analytical Chemists, 74(2), 281-288.Available at: https://doi.org/10.1093/jaoac/74.2.281.

Bekele, J., \& Hassanali, A. (2001). Blend effects in the toxicity of the essential oil constituents of ocimum kilimandscharicum and Ocimum kenyense (Labiateae) on two post-harvest insect pests. Phytochemistry, 57(3), 385-391.Available at: https://doi.org/10.1016/s0031-9422(01)00067-x.

Bosque-Perez, N. A., \& Buddenhagen, I. W. (1992). The development of host-plant resistance to insect pests: Outlook for the tropics. In: Menken, S.B.J., et al. (Ed.). Paper presented at the 8th International Symposium. Insect-pest Relationships. Kluwer Academic Publishers, Dordrech.

Caswell, G. H. (1962). Agricultural entomology in the tropics (pp. 40 -76). London: Edward Arnold.

Chapman, R. (2000). Entomology in the twentieth century. Annual Review of Entomology, 45(1), 261-285.

CIMMYT. (2001). Maize research Highlights 1999-2000. International maize and wheat improvement centre. Mexico: CIMMYT.

Classen, D., Arnason, J., Serratos, J., Lambert, J., Nozzolillo, C., \& Philogene, B. (1990). Correlation of phenolic acid con tent of maize to resistance to Sitophilus zeamais, the maize weevil, in CIMMYT'S collections. Journal of Chemical Ecology, $16(2), 301-315$.

Derera, J., Pixley, K., \& Giga, D. (2001). Resistance of maize to the maize weevil: I. Antibiosis. African Crop Science Journal, 9(2), 431-440.

Dobie, P. (1974). The laboratory assessment of the inherent susceptibility of maize varieties to post-harvest infestation by Sitophilus zeamais Motsch. (Coleoptera, Curculionidae). Journal of Stored Products Research, 10(3-4), 183-197.Available at: https://doi.org/10.1016/0022-474x(74)90006-x.

Dobie, P. (1977). The laboratory assessment of the inherent susceptibility of maize varieties to post-harvest infestation by sitophilus zeamais motsch. Coleoptera, Curculionidae. Journal of Stored Products Research, 1O(3-4), 183-197.Available at: https://doi.org/10.1016/0022-474x(74)90006-x.

FAO. (1991). Maize post-harvest operations. Rome: FAO.

García-Lara, S., Bergvinson, D. J., Burt, A. J., Ramputh, A. I., Díaz-Pontones, D. M., \& Arnason, J. T. (2004). The role of pericarp cell wall components in maize weevil resistance. Crop Science, 44(5), 1546-1552.Available at: https://doi.org/10.2 135/cropsci2004.1546. 
Gwinner, J., Harnisch, R., \& Muck, O. (1996). Manual on the prevention of post-harvest seed losses, post-harvest project (pp. 294-295). Hamburg, FRG: GTZ, D-2000.

Hallauer, A. R., \& Miranda, J. B. (1988). Quantitative genetics in maize breeding Iowa state (pp. 49-52). Ames: University Press.

Kamanula, J., Sileshi, G. W., Belmain, S. R., Sola, P., Mvumi, B. M., Nyirenda, G. K., . . Stevenson, P. C. (2011). Farmers' insect pest management practices and pesticidal plant use in the protection of stored maize and beans in Southern Africa. International Journal of Pest Management, 57(1), 41-49.Available at: https://doi.org/10.1080/09670874.2010.522264.

Keba, T., \& Waktole, S. (2013). Differential resistance of maize varieties to maize weevil (Sitophilus zeamais Motschulsky) (Coleoptera: Curculionidae) under laboratory conditions. Journal of Entomology, 10(1), 1-12.Available at: https://doi.org/10.3923/je.2013.1.12.

Kossou, D. K., Mareck, J. H., \& Bosque-Perez, N. (1993). Comparison of improved and local maize varieties in the republic of Benin with emphasis on susceptibility to sitophilus zeamais motschulsky. Journal of Stored Products Research, 29(4), 333343.

Makate, N. (2010). The susceptibility of different maize varieties to post harvest infestation by Sitophilus zeamais (Motsch) (Coleoptera: Curculionidae). Scientific Research Essay, 5030-5034.

Makkar, H. P. S. (2003). Quantification of Tannis in tree and shrub foliage, laboratory manual. Vienna, Austria: Kluwer Academic Publishers.

Pixley, K. (1997). CIMMYT Mid-altitude maize breeding programme (Vol. 97, pp. 7-13). CIMMYT-Zimbabwe Annual Research Report, 1996/97.

Sen, S., Mukhopadhyay, S., Wetzel, J., \& Biswas, T. K. (1994). Characterization of the mitochondrial DNA polymerase from Saccharomyces cerevisiae. Acta Biochimica Polonica, 41(1), 79-86.

Serratos, J. A., Blanco-Labra, A., Arnason, J. T., \& Mihm, J. A. (1997). Genetics of maize grain resistance to maize weevil. In Mihm J.A. Insect resistant maize: Recent advances and utilization. Paper presented at the Proceedings of an International Symposium, 27 Nov - 3 Dec, 1994.

Serratos, J., Arnason., J., Baum, B., Gale, J., Lambert, J., Bergvinson, D., . . Jewell, D. (1993). Variation in resistance of mexican landraces of maize to maize weevil Sitophilus zeamais, in relation to taxonomic and biochemical parameters. Euphytica, 74(3), 227-236.

Siwale, J., Mbata, K., McRobert, J., \& Lungu, D. (2007). Comparative resistance of improved maize genotypes and landraces to maize weevil. African Crop Science Journal, 17, 1-16.

Tongjura, J. D. C., Amuga, G. A., \& Mafuyai, H. B. (2010). Laboratory assessment of the susceptability of some varieties of Zea mays infested with Sitophilus zeamais, Motsch. Coleoptera, Curculionidae in Jos, Plateau State, Nigeria. Science World Journal, 5(2).Available at: https://doi.org/10.4314/swj.v5i2.61514.

Views and opinions expressed in this article are the views and opinions of the author(s), International Journal of Sustainable Agricultural Research shall not be responsible or answerable for any loss, damage or liability etc. caused in relation to/arising out of the use of the content. 